\title{
An efficient Data Packet Loss Regulator Using Tokens in IoT Networks
}

\author{
Ch. Lakshmi Kumari
}

1Information Technology,MGIT, Hyderabad, Telangana, India

To Cite this Article

Ch. Lakshmi Kumari, "An efficient Data Packet Loss Regulator Using Tokens in IoT Networks", International Journal for Modern Trends in Science and Technology, 6(8S): 108-111, 2020.

\section{Article Info}

Received on 16-July-2020, Revised on 15-August-2020, Accepted on 25-August-2020, Published on 28-August-2020.

\section{ABSTRACT}

Presently the Internet put up concurrent audio, video, and data traffic. This requires the Internet to assurance the packet loss which at its turn depends very much on congestion control. A series of protocols have been presented to enhancement the insufficient TCP mechanism controlling the network congestion. CSFQ was designed as an open-loop controller to provide the fair best effort service for supervising the per-flow bandwidth consumption and has become helpless when the P2P flows started to dominate the traffic of the Internet. Token-Based Congestion Control (TBCC) is based on a closed-loop congestion control opinion, which limits token resources consumed by an end-user and provides the fair best effort service with $O(1)$ complexity. As Self-Verifying CSFQ and Re-feedback, it experiences a heavy load by policing inter-domain traffic for lack of trust. In this paper, Stable Token-Limited Congestion Control (STLCC) is introduced as new protocols which appends inter-domain congestion control to TBCC and make the congestion control system to be stable. STLCC is able to shape output and input traffic at the inter-domain link with $O(1)$ complexity. STLCC produces a congestion index, pushes the packet loss to the network edge and improves the network performance. Finally, the simple version of STLCC is introduced. This version is deployable in the Internet without any IP protocols modifications and preserves also the packet datagram.

KEYWORDS: packet loss, p2p, token, protocols

\section{INTRODUCTION}

Modern IP network facilities provide for the simultaneous digital transmission of voice, video, and data. These services require congestion control protocols and algorithms which can solve the packet loss limitation can be kept under control. Congestion control is therefore, the cornerstone of packet switching networks. It should prevent congestion collapse, provide fairness to competing flows and optimize transport performance indexes such as throughput, delay and loss.

The literature abounds in papers on this subject there are paper son high-level models of the flow of packets through the network, and on specific network architecture. Despite this vast literature, congestion control in telecommunication networks fights with two major problems that are not totally solved. The first one is the time-varying delay between the control point and the traffic sources. The second one is related to the possibility that the traffic sources do not follow the feedback signal. This latter may happen because some sources are silent as they have nothing to transmit. Congestion control of the best-effort service in the Internet was originally designed for a cooperative environment. It is still mainly dependent on the TCP congestion 
control algorithm at terminals, supplemented with load shedding at congestion links. This model is called the Terminal Dependent Congestion Control case.

In the existing system, the sender sends the packets without the intermediate station. The data packets has been losses many and

time is wasted. Retransmission of data packets is trouble.

Internet provides simultaneous audio, video, and data traffic. This is possible when the Internet guarantees the packet loss which depends very much on congestion control. A series of protocols have been introduced for controlling the network congestion [2]. Modern IP network services provide for the simultaneous digital transmission of voice, video, and data. These services require congestion control protocols and algorithms which can solve the packet loss parameter can be kept under control. Congestion control is therefore, the cornerstone of packet switching networks. It should prevent congestion collapse [9], provide fairness to competing flows and optimize [16] transport performance indexes such as throughput, delay and loss

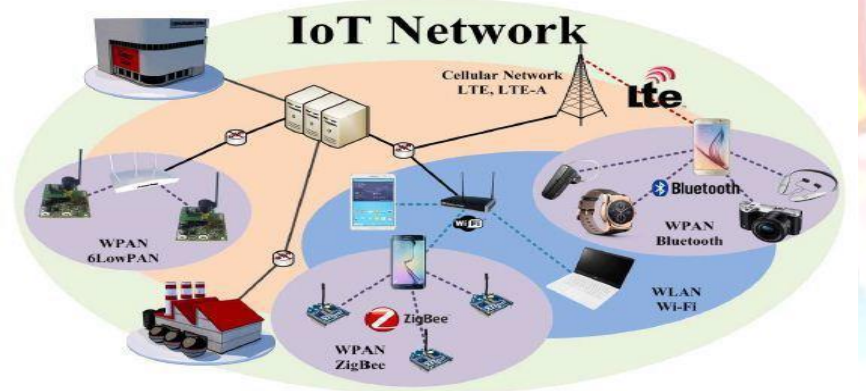

Simplified block diagram of basic building block of IoT is shown in the figure 2 . In this IoT devices accommodate many sensors and those sensors are associated with the IoT area networks that assembles the packet containing signals from these sensors. The data chunks collected by these sensors are processed by the processor and transferred to gateways over the IoT area networks, which are configured using several transmission technologies of which majority are wireless network technologies. Some of the technologies which rule in IoT network are wireless Personal Area network [WPAN]. This WPAN includes network like ZigBee, Bluetooth, and Ipv6 over low power wireless personal Area Network etc. On a slightly larger wireless network area scale, WLAN (Wireless Local Area Network) which includes Wi-Fi is to be used. On a larger scale, the mobile communication technologies like $2 \mathrm{G}, 3 \mathrm{G}, 4 \mathrm{G}$, LTE remains. Smartphones and mobile communication system will be used and they will connect to the base stations and base stations will provide connectivity to the Wide Area Network (WAN) which is the Internet

\section{II.PROPOSED SYSTEM}

In the present system, the sender sends the packets without the intermediate station. The data packets has been losses many and time is wasted. Retransmission of data packets is trouble. Modern IP network facilities provide for the concurrent digital transmission of voice, video, and data. These services require congestion control protocols and algorithms which can solve the packet loss parameter can be kept under control. Congestion control is therefore, the cornerstone of packet switching networks. It should prevent congestion failure, provide equality to competing flows and optimize transport performance indexes such as throughput, delay and loss. The literature abounds in papers on this subject; there are papers on high-level models of the flow of packets through the network, and on specific network architecture. In this paper a new and better mechanism for congestion control with application to Packet Loss in networks with $\mathrm{P} 2 \mathrm{P}$ traffic is planned. In this new method the edge and the central routers will write a measure of the quality of service guaranteed by the router by writing a digital number in the Option Field of the datagram of the packet. This is called a token. The token is read by the path routers and interpreted as its value will give a measure of the bottleneck especially at the edge routers. Based on the token number the edge router at the source, thus reducing the congestion on the path.

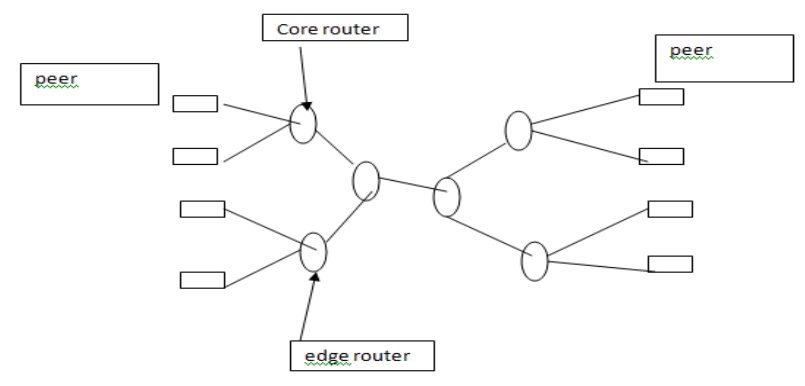

A central router is a router designed to operate in the Internet Backbone or central. To achieve this role, a router must be able to support many telecommunications interfaces of the highest speed in use in the core Internet and must be able to forward IP packets at full speed on all of them. It must also support the routing protocols being used in the core. A core router is distinct from edge routers.Edge routers sit at the edge of a backbone 
network and connect to core routers. The token is read by the path routers and interpreted as its value will give a measure of the congestion especially at the edge routers. Based on the token number the edge router at the source, thus reducing the congestion on the path.

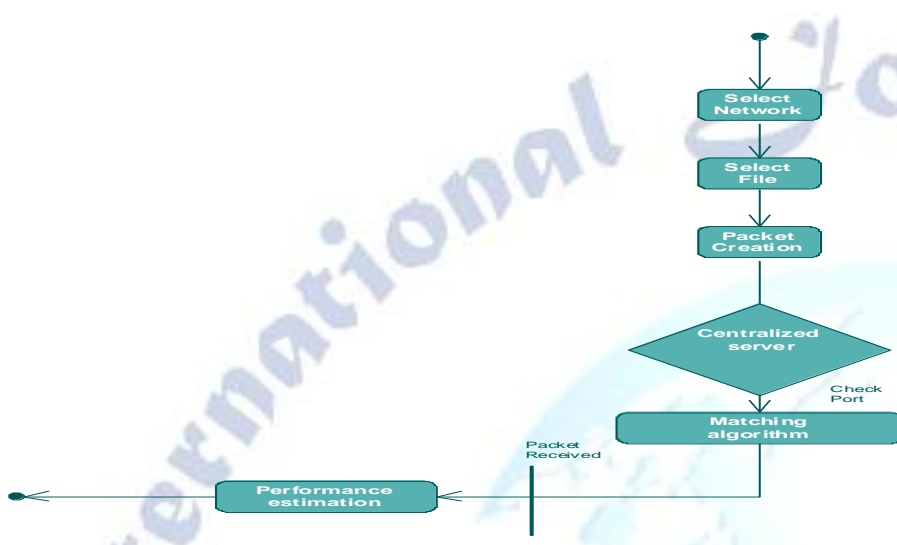

Fig1:system architecture

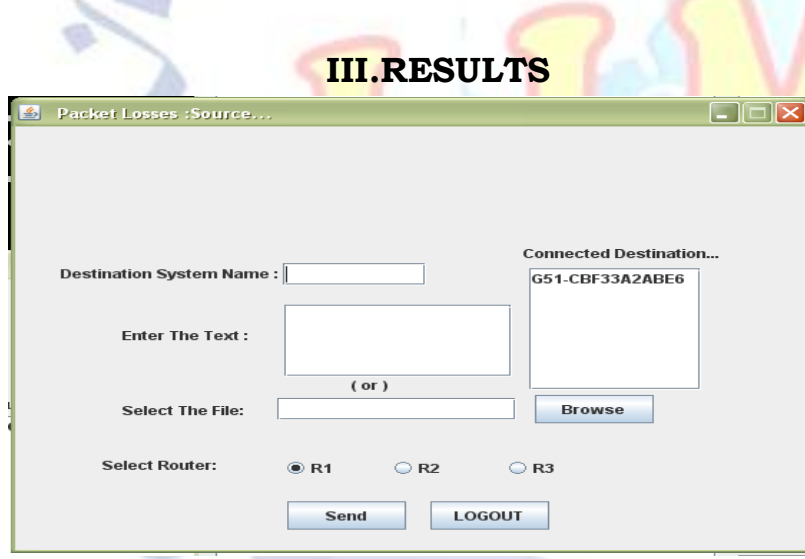

Fig2:selecting network

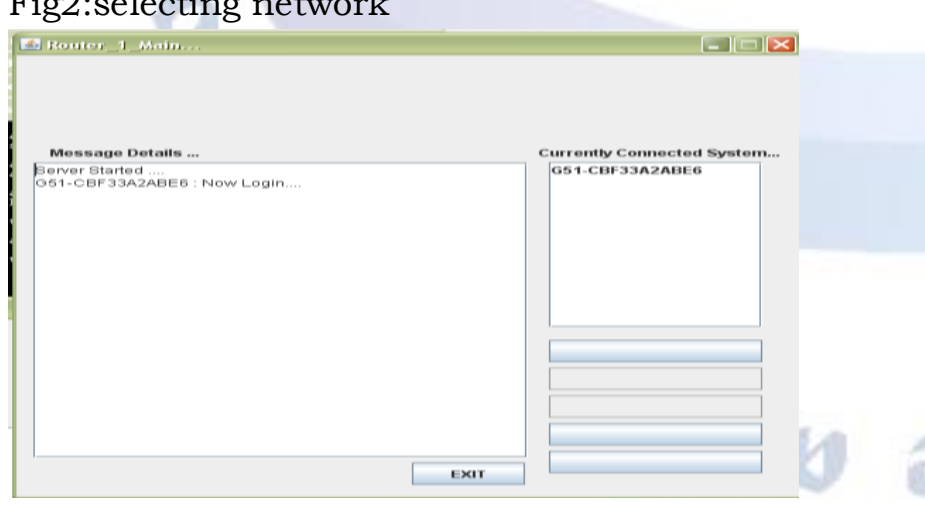

Fig3:connecting to server

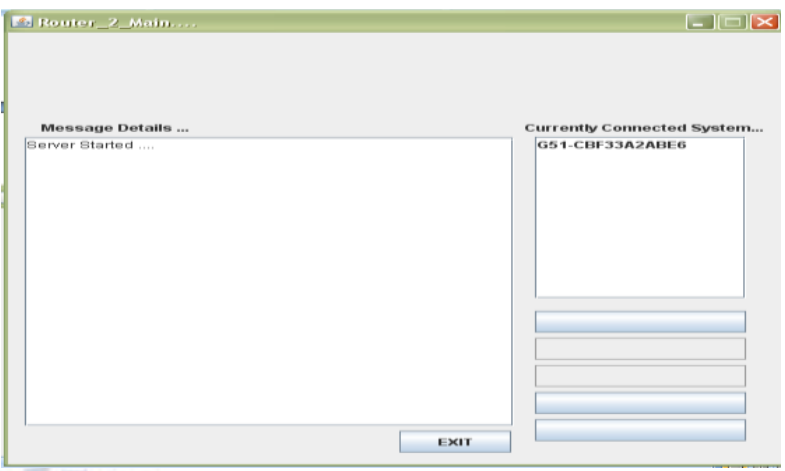

Fig4:server started

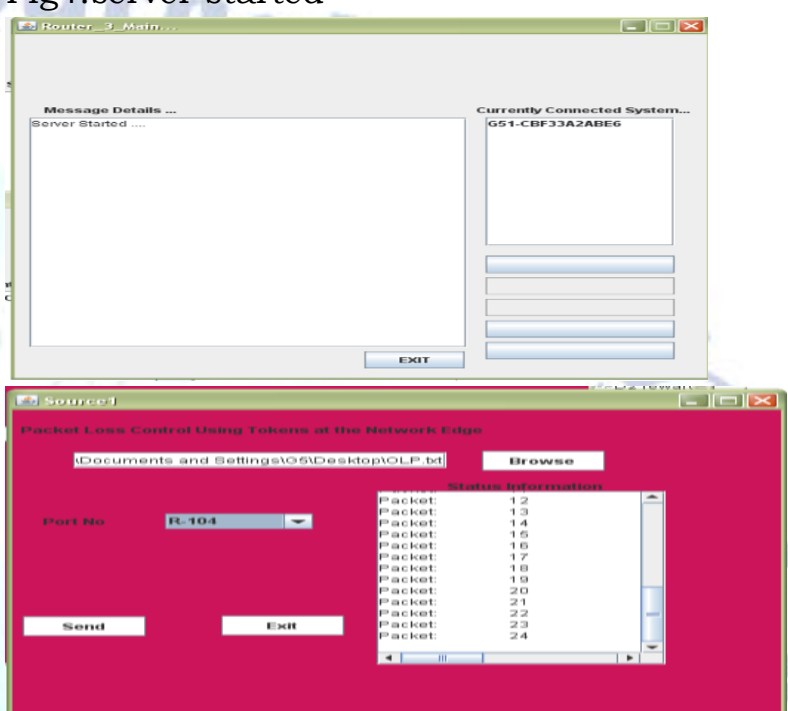

Fig6:packet loss control at network edge

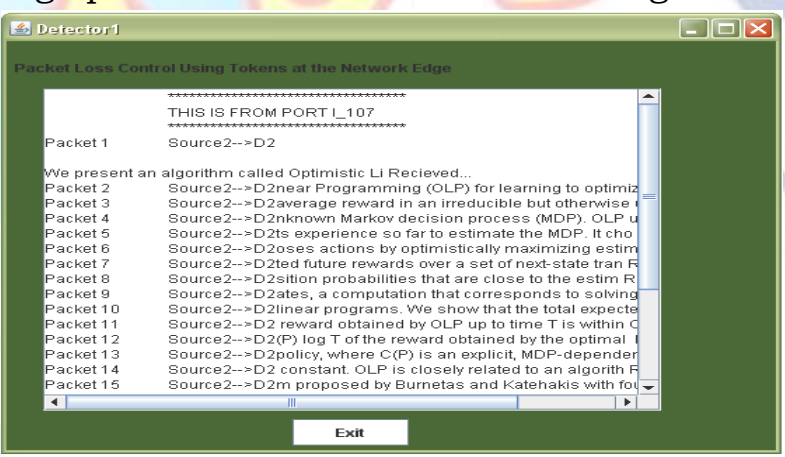

Fig7:port 1_107 information

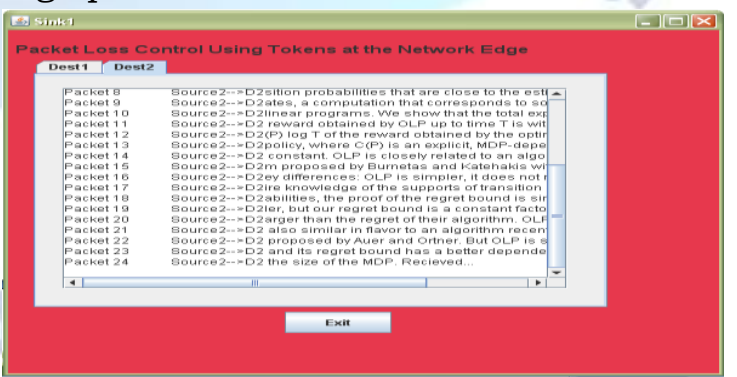

Fig8:source to destination informatiom

\section{VI.CONCLUSION}

The "packet loss control using tokens at network edge" has been successfully completed. The information is sent without loss of and packet and errorfree. This project is organized as follows. The architecture of Token-Based Congestion Control, 
which provides fair bandwidth allocation to end-users in the same domainwill be introduced. It evaluates two congestion control algorithms CSFQ and TBCC. STLCC is presented and the simulation is designed to demonstrate its validity. The Unified Congestion Control Model which is the abstract model of CSFQ, Re-feedback and STLCC.

Finally, conclusions will be given. To inter-connect two TBCC domains, the inter-domain router is added to the TBCC system. To support the SKA arrangement, the inter-domain router should limit its output token rate to the rate of the other domains and police the incoming token rate from peer domains.

\section{ACKNOWLEDGMent}

This research was supported by my institution, departmentand my colleaguesgues who supported my paper. We are thankful to our colleagues who provided expertise that greatly.

\section{REFERENCES}

[1] Andrew S.Tanenbaum, Computer Networks, Prentice-Hall International.

[2] Ministry of Internal Affairs and Communication, Panan, "White paper 2016: Information and Communication", 2016

[3] T. Yokotani, "Tutorial: M2M/IoT Technical Overview and its Standardization", APNOMS 2016, 2016

[4] T. Fujita, Y. Goto, A. Koike, "M2M Architecture trend and technical issues" The Journal of Institute of Electronics, Information and Communication Engineer, Vol.96, No.3, pp.305- 312, 2013

[5] S. Floyd and V. Jacobson. Random Early Detection Gateways for Congestion Avoidance, ACM/IEEE Transactions on Networking. Zhiqiang Shi, Token-based congestion control: Achieving fair resource allocations in P2P networks, ITU-T Kaleidoscope Academic Conference.

[6] Ion Stoica, Scott Shenker, Hui Zhang, "Core-Stateless Fair Queueing: A Scalable Architecture to Approximate Fair Bandwidth Allocations in High Speed Networks".

[7] D. Qiu and R. Srikant. Modeling and performance analysis of BitTorrent- like peer-to-peer networks. In Proc. of SIGCOMM, 2004.

[8] Floyd, S., Connections with Multiple Congested Gateways in Packet- Switched Networks Part 1: One-way Traffic. Computer Communications Review, Vol.21, No.5, October 1991, p. 30-47.

[9] J.C.R. Bennett, D.C. Stephens, and H. Zhang. High speed, scalable, and accurate implementation of packet fair queueing algorithms in ATM networks. In Proceedings of IEEE ICNP '97, pages 7-14, Atlanta, GA, October 1997

[10] J. Jones. (1991, May 10). Networks (2nd ed.) [Online]. Available: http://www.atm.com

[11] (Journal Online Sources style) K. Author. (year, month). Title. Journal [Type of medium]. Volume(issue), paging if given. Available: http://www.(URL

[12] R. J. Vidmar. (1992, August). On the use of atmospheric plasmas as electromagnetic reflectors. IEEE Trans. Plasma Sci. [Online]. 21(3). pp. 876-880. Available: http://www.halcyon.com/pub/journals/21ps03-vidmar 